\title{
Subsecond Spiral CT
}

National Cancer Institute

\section{Source}

National Cancer Institute. Subsecond Spiral CT. NCI Thesaurus. Code C19912.

A type of computer tomography that allows a full rotation of the X-ray tube around the patient in less than 1 second. 